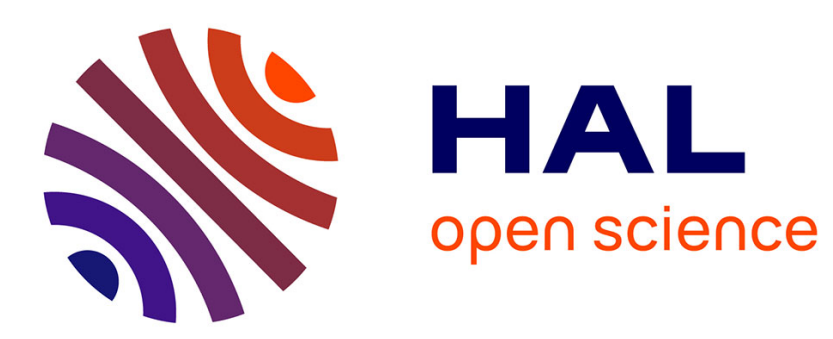

\title{
GPU Environmental Delegation of Agent Perceptions: Application to Reynolds's Boids
}

\author{
Emmanuel Hermellin, Fabien Michel
}

\section{To cite this version:}

Emmanuel Hermellin, Fabien Michel. GPU Environmental Delegation of Agent Perceptions: Application to Reynolds's Boids. MABS: Multi-Agent Based Simulation, May 2015, Istanbul, Turkey. pp.71-86, 10.1007/978-3-319-31447-1_5 . lirmm-01284864

\section{HAL Id: lirmm-01284864 https://hal-lirmm.ccsd.cnrs.fr/lirmm-01284864}

Submitted on 8 Mar 2016

HAL is a multi-disciplinary open access archive for the deposit and dissemination of scientific research documents, whether they are published or not. The documents may come from teaching and research institutions in France or abroad, or from public or private research centers.
L'archive ouverte pluridisciplinaire HAL, est destinée au dépôt et à la diffusion de documents scientifiques de niveau recherche, publiés ou non, émanant des établissements d'enseignement et de recherche français ou étrangers, des laboratoires publics ou privés. 


\title{
GPU Environmental Delegation of Agent Perceptions: Application to Reynolds's Boids
}

\author{
Emmanuel Hermellin, Fabien Michel \\ LIRMM - CNRS - University of Montpellier, 161 rue Ada, 34095 Montpellier, France \\ \{emmanuel.hermellin, fmichel\}@lirmm.fr,
}

\begin{abstract}
Using Multi-Agent Based Simulation (MABS), computing resources requirements often limit the extent to which a model could be experimented with. Regarding this issue, some research works propose to use the General-Purpose Computing on Graphics Processing Units (GPGPU) technology. GPGPU allows to use the massively parallel architecture of graphic cards to perform general-purpose computing with huge speedups. Still, GPGPU requires the underlying program to be compliant with the specific architecture of GPU devices, which is very constraining. Especially, it turns out that doing MABS using GPGPU is very challenging because converting Agent Based Models (ABM) accordingly is a very difficult task. In this context, the GPU Environmental Delegation of Agent Perceptions principle has been proposed to ease the use of GPGPU for MABS. This principle consists in making a clear separation between the agent behaviors, managed by the CPU, and environmental dynamics, handled by the GPU. For now, this principle has shown good results, but only on one single case study. In this paper, we further trial this principle by testing its feasibility and genericness on a classic ABM, namely Reynolds's boids. To this end, we first review existing boids implementations to then propose our own benchmark model. The paper then shows that applying GPU delegation not only speeds up boids simulations but also produces an ABM which is easy to understand, thanks to a clear separation of concerns.
\end{abstract}

Keywords: Multi-Agent Based Simulation, Flocking, GPGPU, CUDA

\section{Introduction}

Because Multi-Agent Based Simulation (MABS) can be composed of many interacting entities, studying their properties using digital simulation may require a lot of computing resources. To deal with this issue, the use of General-Purpose computing on Graphics Processing Units (GPGPU) can drastically speed up simulation runs for a cheap cost [8]. GPGPU relies on using the massively parallel architectures of usual graphic cards to perform general-purpose computing. However, this technology implies a very specific programming scheme which requires advanced GPU (Graphics Processing Unit) programming skills [11].

Because there are many different MAS (Multi-Agent Systems) models, there is no generic way for implementing MAS using GPGPU. It is not about only 
changing of programming language. With GPGPU, many concepts which are present in sequential programming are no longer available. Especially, important features of object-oriented languages simply cannot be used (inheritance, composition, etc.). So, it is very difficult to adapt an Agent Based Model (ABM) so that it can be run on the GPU. Considering this issue, hybrid systems represent an attractive solution. Because the execution of the MAS is shared between the Central Processing Unit (CPU) and the GPU, it is thus possible to select only what is going to be translated and executed by the graphics card.

In this paper, we propose to challenge the feasibility and interest of the $G P U$ Environmental Delegation of Agent Perceptions (GPU Delegation for short) principle which is based on an hybrid approach. This principle consists in identifying and delegating to the environment some of the computations made by the agents. A case study is presented in [9] and shows good results in terms of performances, accessibility and reusability. We propose to trial this principle by using it on a classic ABM, namely Reynolds's boids [14].

In Sect. 2 we review how Reynolds's Boids is implemented in several MABS platforms and then propose our own flocking model in Sect. 3. Section 4 presents the GPU Environmental Delegation of Agent Perceptions principle. In Sect. 5, we describe the implementation of our model and how we have applied $G P U$ Delegation on it. In Sect. 6, we present and discuss the results of our tests. Finally, we conclude and present perspectives in Sect. 7.

\section{Reynolds's Boids}

\subsection{Original Model Overview}

Reynolds was interested in achieving a believable animation of a flock of artificial birds and remarked that it was not possible to use a scripted flock motion. That is why Reynolds proposed an ABM based on local individual rules, namely boids [14]. Reynolds's idea was that boids have to be influenced by the others to flock in a coherent manner: "Boid behavior is dependant not only on internal state but also on external state.".

Reynolds proposes that each agent is subjected to forces that make it move by taking into account the interactions with the others. So, each entity has to follow three behavior rules:

- R.1 Collision Avoidance: Avoid collisions with nearby flockmates

- R.2 Flock Centering: Attempt to stay close to nearby flockmates

- R.3 Velocity matching: Attempt to match velocity with nearby flockmates

Reynolds's boids is recognized as one of the most representative ABM and many agent-based platforms integrate their own boids model.

\subsection{Boids in Current MABS Platforms}

In this section, we compare several implementations of Reynolds's boids that we can find in popular MABS platforms. Among the related works found, we 
only introduce models we were able to download and try with an open source code: NetLogo, StarLogo, Gama, Mason and Flame GPU. For each model, we describe how the three rules are implemented (Collision Avoidance (R.1), Flock Centering (R.2), Velocity matching(R.3)).

NetLogo In NetLogo ${ }^{1}$ [17], all the agents move and try to get closer to their peers. If the distance between them and the nearest neighbor is too small, the agent tries to get away (avoid collision (R.1)), otherwise the agent aligns with its neighbors (R.2). However, there is no speed management (R.3): All the agents have the same velocity during the entire simulation.

StarLogo In StarLogo ${ }^{2}$ [13], the agent searches for his closest neighbor. If the distance to his peer is too small, then the agent turns and gets away to avoid collision (R.1). Otherwise, it moves toward him and use his direction. The search for cohesion (R.2) is not explicitly expressed and the velocity of the agents is fixed throughout the simulation (R.3).

Gama In Gama ${ }^{3}$ [3], agents first look for a virtual target to follow (a moving object in the environment that initiates the flocking behavior). Once the agents have a target, they move according to three functions that implement Reynolds's rules: A separation function to avoid collision (R.1), a cohesion function (R.2) and an alignment function for speed and direction (R.3). The model differs from Reynolds's because the agents need a target to actually make the flocking.

MasOn $\mathrm{MasOn}^{4}[7]$ uses the computation of several vectors to integrate R.1 and R.2. Each agent computes a motion vector composed of an avoidance vector (this is computed as the sum, over all neighbors, of a vector to get away from the neighbors (R.1)), a cohesion vector (this is computed as the sum, over all live neighbors, of a vector towards the "center of mass" of nearby flockers), a momentum vector (a vector in the direction the flocker went last time), a coherence vector (this is computed as the sum, over all live neighbors, of the direction of other flockers are going (R.2)), and a random vector. The velocity is not managed in this model (R.3).

Flame GPU Flame GPU ${ }^{5}[15]$ is the only GPGPU implementation that we were able to test. In this model, R.1 R.2 and R.3 are actually implemented into three independent functions.

\footnotetext{
${ }^{1}$ https://ccl.northwestern.edu/netlogo/

${ }^{2}$ http://education.mit.edu/starlogo/

3 https://code.google.com/p/gama-platform/

${ }^{4}$ http://cs.gmu.edu/ eclab/projects/mason/

${ }^{5}$ http://www.flamegpu.com/
} 
Summary Table 1 summarizes the implementations of Reynolds's rules, sets out the main features of the models and gives performance informations.

Performances We evaluate for each model the average computation time in milliseconds for an iteration. The purpose of this evaluation is to give an idea of the possibilities of each implementation. So, we use as common parameter an environment of $512 \times 512$ containing 4000 agents. Our test configuration is composed of an Intel i7-4770 processor (Haswell generation, 3.40 GHz) and an Nvidia K4000 graphics card (768 CUDA cores).

It has to be noted that for StarLogo, we observed a computation time higher than a second from 400 simulated agents so that we did not push the tests further. Finally, for Flame GPU, it was not possible to modify the number of agents in the simulation which is of 2048 .

Table 1. Boids in common MABS platforms

\begin{tabular}{|c|c|c|c|c|c|}
\hline Platform & \begin{tabular}{|} 
Complian \\
Collision R.1
\end{tabular} & $\begin{array}{l}\text { nce with Rey } \\
\text { Model } \\
\text { Cohesion R.2 }\end{array}$ & $\begin{array}{l}\text { ynolds's } \\
\text { Velocity R.3 }\end{array}$ & Main characteristics & Performances \\
\hline NetLogo & $\mathrm{X}$ & $\mathrm{X}$ & & $\begin{array}{l}\text { R.3 is not implemented: Velocity is fixed } \\
\text { throughout the simulation }\end{array}$ & $214 \mathrm{~ms}(\mathrm{CPU} / \mathrm{Logo})$ \\
\hline StarLogo & $\mathrm{X}$ & & & $\begin{array}{l}\text { A minimalist implementation of behavior } \\
\text { rules (only the collision avoidance is im- } \\
\text { plemented) }\end{array}$ & *1000 ms (CPU / Logo) \\
\hline Gama & $\mathrm{X}$ & $\mathrm{X}$ & $\mathrm{X}$ & $\begin{array}{l}\text { Flocking behaviour when agents have a } \\
\text { target to follow }\end{array}$ & $375 \mathrm{~ms}(\mathrm{CPU} / \mathrm{GAML})$ \\
\hline MasOn & $\mathrm{X}$ & $\mathrm{X}$ & & $\begin{array}{l}\text { The rules R.1 and R.2 are reinterpreted } \\
\text { into a global vector with addition of ran- } \\
\text { dom components, no speed management }\end{array}$ & $45 \mathrm{~ms}$ (CPU / Java) \\
\hline Flame GPU & $\mathrm{X}$ & $\mathrm{X}$ & $\mathrm{X}$ & The three rules are explicitly implemented & *82ms (GPU / C,XML) \\
\hline
\end{tabular}

\section{Reynolds's Boids: Our Model and Implementation}

From the previous study, we notice disparities between the various presented models. Indeed, Reynolds's rules allow a large variety of interpretations. For instance, we notice that the speed adaptation rule (R.3) is not always taken into account compared to R.1 and R.2 which are implemented in almost every model (except StarLogo). However, when R.3 is implemented, the collective behavior becomes much more convincing in terms of flocking dynamics and movement believability. Also, in some works, alignment and cohesion behaviors are merged. The models clarifying the difference between this two behaviors offer more interesting movements. 
The model that we propose takes into account the points observed previously: Overall, when the three rules are explicitly considered, the dynamics and the movement of the agents are more convincing. So, our model integrates R.1, R.2 and R.3 and also follows the KISS (Keep It Simple and Stupid) principle in the aim of creating a minimalist version (with as few parameters as possible). So the model focuses only on the speed and the orientation of the agent ${ }^{6}$.

Each entity has a global behavior which consists in moving while adapting its speed and direction. To this end, the proximity with the other agents is first tested and then Reynolds's rules are triggered accordingly. More specifically, every agent first looks in its vicinity. If no agent is present, then it continues to move in the same direction. Otherwise, the agent checks if the neighbors are not too close. Depending on the proximity between entities, agents separate (R.1), align with other entities or create cohesion (R.2). Then agents adapt their speed (R.3), move and restart the process. Figure 1 summarizes the global behavior process.

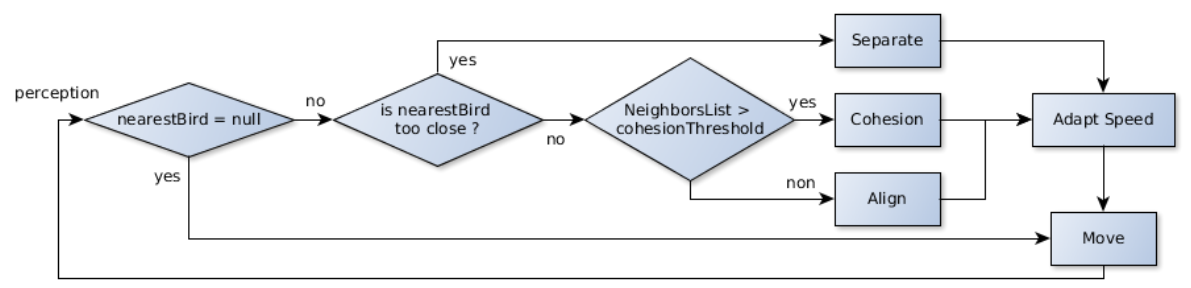

Fig. 1. Flocking: Global behavior process

In our model, we have two types of parameters: 5 constants for the model and 3 attributes specific to each agent. The constants are the following ones:

- fieldOfView (agent's field of view);

- minimalSeparationDistance (minimum distance between agents);

- cohesionThreshold (necessary number of agents to begin cohesion);

- maximumSpeed (maximum speed of the agent);

- maximumRotation (maximum angle of rotation).

The attributes specific to each agent are the following ones:

- heading (agent's heading);

- velocity (agent's speed);

- nearestNeighborsList (the list containing nearest neighbors).

\footnotetext{
${ }^{6}$ The orientation is an angle in degree (between 0 and 360) which gives the heading of the agent according to the landmark fixed in the environment.
} 
Separation Behavior R.1 When an agent is too close from another one, it separates (R.1). This behavior consists in retrieving the heading of both agents. If these two directions lead to a collision, agent rotates to avoid its neighbor (see Algorithm 1).

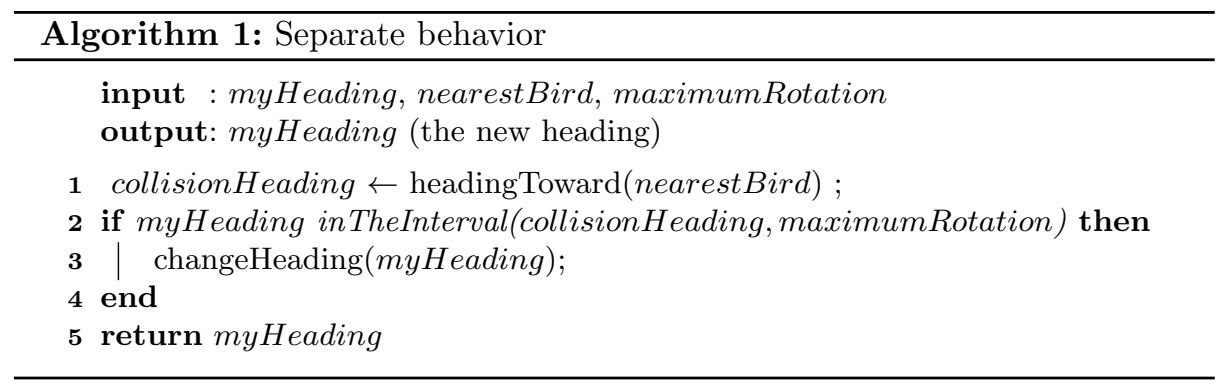

Align Behavior R.2 When an agent comes closer to other entities, it tries to align itself with them, by adjusting his direction according to its nearest neighbor (see Algorithm 2).

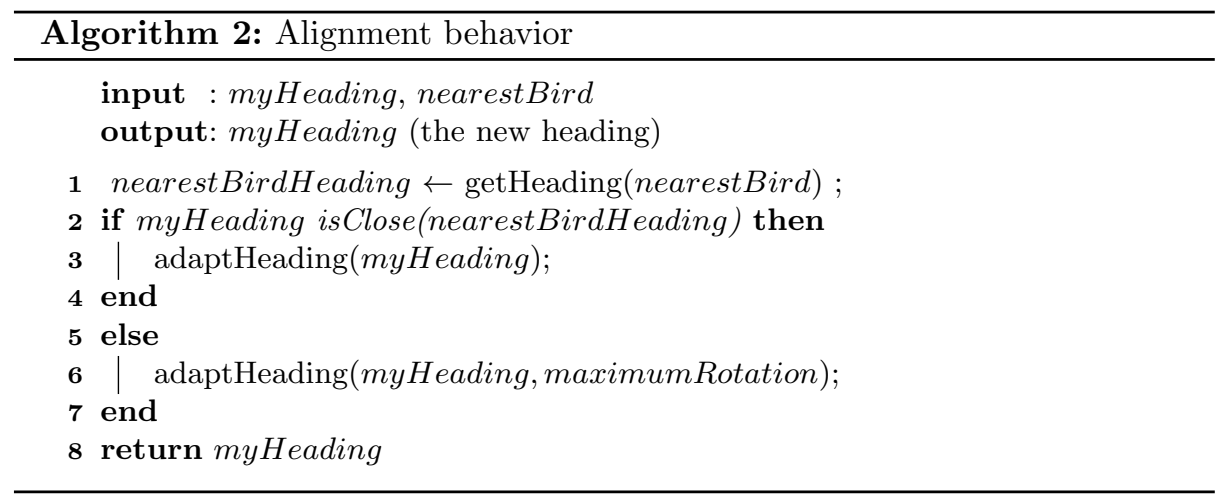

Cohesion Behaviors R.2 When multiple agents are close to each other without having to separate, they have a cohesion behavior. Each agent retrieves the directions of its neighbors and adjusts its own direction based on the average direction found, thus strengthening the cohesion of the group (see Algorithm 3).

Speed Adaptation R.3 Before moving, the agents adapt their speed (R.3). During all the simulation, every agent modifies its speed according to that of 


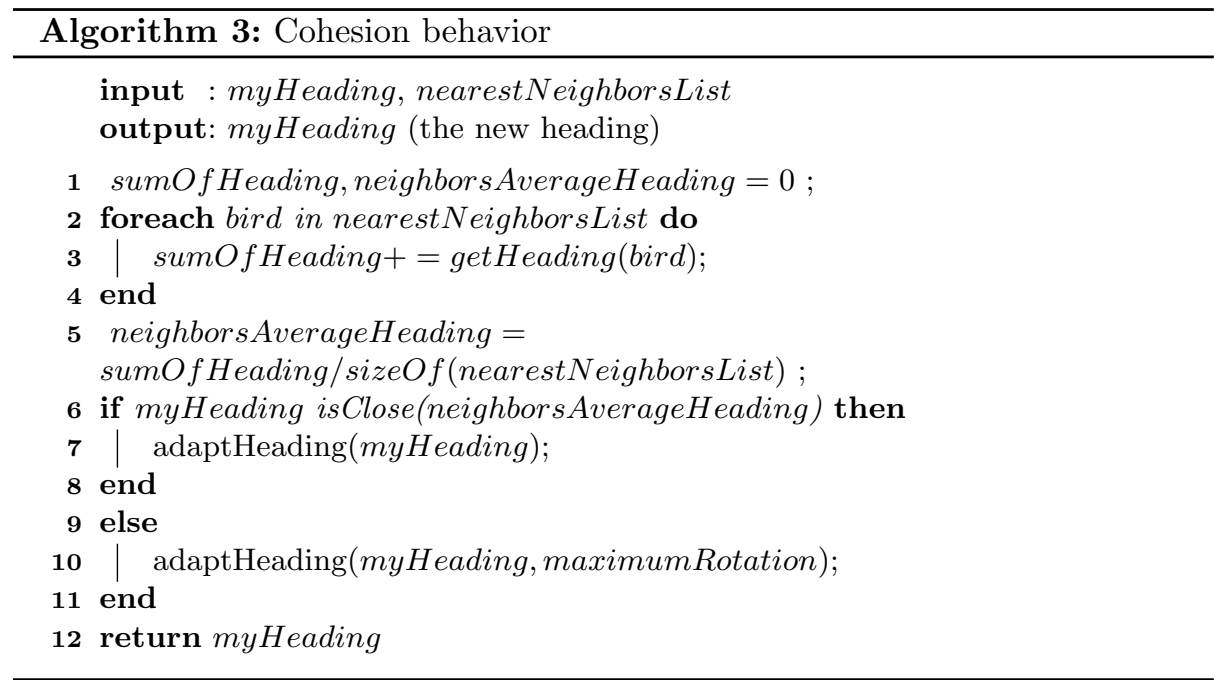

its neighbors. If the agent has just executed the behavior of separation (R.1), it accelerates to get free more quickly. Otherwise, the agent adjusts its speed to make it correspond to that of its neighbors (in the limit authorized by the maximumSpeed constant).

Testing Our Model We have put online a set of videos that show our model in action ${ }^{7}$. On this page are also available the source codes of the mentioned models and the resources required to test our solution.

\section{GPU Environmental Delegation of Agent Perceptions}

\subsection{MABS and GPGPU}

GPGPU Basics For the purpose of understanding the basics of GPGPU programming, one has to have in mind that it is strongly connected to the underlying hardware architecture of GPU. In this respect, one of the main differences between a CPU and a GPU is the number of processing cores which is far more important in the GPU case.

Today, GPU are composed of hundreds or thousands of processing core (grouped into Streaming Multiprocessors, SM) forming a highly parallel structure able to perform more varied computing. GPGPU relies on using the SIMD (Single Instruction, Multiple Data) parallel model. Also called stream processing, the underlying programming approach consists in performing the same operation on multiple data points simultaneously. In other words, GPGPU relies on

\footnotetext{
$\overline{7}$ www.lirmm.fr/ hermellin/Website/Reynolds_Boids_With_TurtleKit.html
} 
the simultaneous execution of a series of computations (kernels) on a data set (the flow - stream).

The related programming models rely on the following work flow: The CPU is called the host and plays the role of scheduler. The host manages data and triggers kernels, which are functions specifically designed to be executed by the GPU, which is called the device. The GPU part of the code really differs from sequential code and has to fit the underlying hardware architecture. More precisely, the GPU device is programmed to proceed the parallel execution of the same procedure, the kernel, by means of numerous threads. These threads are organized in blocks (the parameters blockDim.x, blockDim.y characterize the size of these blocks), which are themselves structured in a global grid of blocks. Each thread has unique 3D coordinates (threadIdx.x, threadIdx.y, threadIdx.z) that specifies its location within a block. Similarly, each block also has three spatial coordinates (respectively blockIdx.x, blockIdx.y, blockIdx.z) that localize it in the global grid. Figure 2 illustrates this organization for the 2D case. So, each thread works with the same kernel but uses different data according to its spatial location within the $\operatorname{grid}^{8}$. Moreover, each block has a limited thread capacity according to the hardware in use.

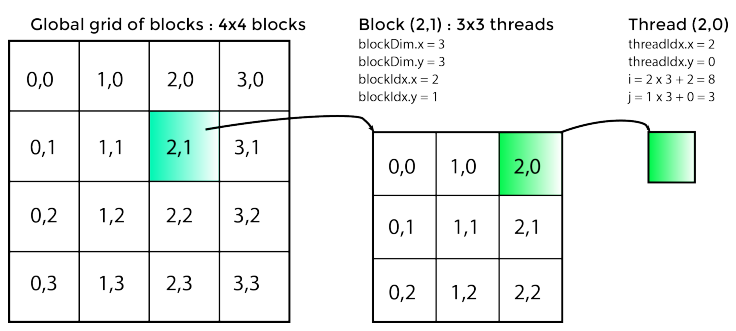

Fig. 2. Thread, blocks, grid organization

So, a multithreaded program is partitioned into blocks of threads that execute independently from each other. The distribution of blocks and threads on SM may be automatic and is provided by the runtime and drivers. For instance, in the GPGPU platform from Nvidia (Compute Unified Device Architecture, CUDA), a CUDA program can be executed on any number of multiprocessors as illustrated by Fig. 3, and only the runtime system needs to know the physical multiprocessor count.

Implementing MABS Using GPGPU There are two ways of implementing a model on GPU: (1) all-in-GPU, for which the simulation runs entirely on the graphics card and (2) hybrid, the execution of the simulation is shared between

\footnotetext{
${ }^{8}$ In this context, Thread is similar to the concept of task: A thread may be considered as an instance of the kernel which is performed on a restricted portion of the data depending on its location in the global grid (its identifier)
} 


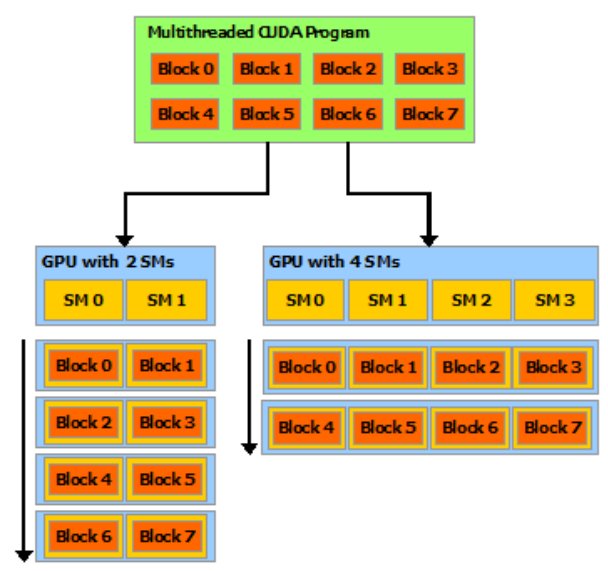

Fig. 3. Automatic Scalability (Source: Nvidia programming guide)

the CPU and the GPU. In the first case (1), it is not trivial to take an existing model and translate it to make it work on GPU. GPU are very restrictive in operations and programming and the hardware can only be used in certain ways that requires advanced GPU programming skills. The hybrid approach (2) allows to use jointly the CPU and GPU and thus has two major advantages. Firstly, it brings more flexibility because one can choose what is going to be executed on the GPU, thus providing greater accessibility to the developed tools (as clearly shown in [6] [5], [16], [18]). Secondly, as hybrid systems are modular by design, they make it possible to use agents with complex and heterogeneous architectures. The GPU Environmental Delegation of Agent Perceptions principle relies on an hybrid approach.

\subsection{Converting Agent Perceptions in Environmental Dynamics}

The Principle GPU Environmental Delegation of Agent Perceptions principle was proposed in [9]. This principle consists in making a clear separation between the agent behaviors, managed by the CPU, and environmental dynamics, handled by the GPU. The underlying idea is to identify in the behavior of the agents some computations which can be transformed into environmental dynamics. It has been first stated as follows: Any agent perception computation not involving the agents state could be translated to an endogeneous dynamic of the environment, and thus considered as a potential GPU environment module.

Related Works GPU delegation has to be related to other works which reify parts of the agents' computations in structures related to other concepts such as the interactions or the environment.

In the MABS context, the EASS (Environment As Active Support for Simulation)[1] approach aims at strengthening the role of the environment by delegat- 
ing to it the scheduling policy and adding a filtering system for the perceptions. IODA (Interaction Oriented Design of Agent simulations) [4] is centered on the notion of interaction and considers that agent behaviors can be described in a abstract way as a rule called interaction. [12] proposes to reduce the complexity of the models by using an environment-centered approach: Some agent interaction patterns are modeled as environmental dynamics to ease the reusability and the integration of various agent processes.

GPU Delegation on a Case Study The integration of GPU computations was performed in TurtleKit ${ }^{9}$ [10]. TurtleKit is a generic spatial ABM, implemented with Java, wherein agents evolve in a $2 \mathrm{D}$ environment discretized in cells. The proposed hybrid approach integrated in TurtleKit focuses on modularity. In this context, this allows to achieve three objectives: (1) maintain accessibility in the agent model while using GPGPU, (2) to scale and work with a large number of agents on large environment sizes and (3) promote re-usability in the particular context of GPU programming.

$G P U$ Delegation has been used only once on a model of multi-level emergence (MLE) [2] of complex structures in TurtleKit. This very simple model relies on a unique behavior which allows to generate complex structures which repeat in a fractale way. The agent behavior is extremely simple and is based on the perception, the spread and the reaction to pheromones. So, in these works, GPU modules dedicated to the perception and the spread of pheromones were proposed.

\section{GPU Delegation for Boids}

\subsection{Applying GPU Delegation}

With respect to the underlying hybrid approach, GPU Delegation is about identifying specific behaviors which can be turned into environmental dynamics. Especially, GPU Delegation states that agent perception computations that do not involve the agents state could be translated into environmental dynamics. For instance, in the previous case study (MLE), the computation related to the agent perceptions used to decide how the agents move according to pheromones (i.e. following gradients) is completely independent from the agents' state: Gradients are equals whatever the agents' states. So a GPU module (environmental dynamics) has been produced for computing pheromones gradients independently from the agents.

In our flocking model, it has not been possible to find a computation which is independent from the agents' attributes. However, we actually identified some computations that can be done without modifying the agent's state so that they can be thus translated into an environmental dynamics computed by the GPU.

\footnotetext{
$\overline{9}$ http://www.turtlekit.org
} 
Indeed, the cohesion behavior consists in averaging the orientations of neighboring agents according to the selected FieldOfView ${ }^{10}$. All the agents perform this computation in their own behavior and use the result to adapt their direction. The sequential implementation of this process is as follows:

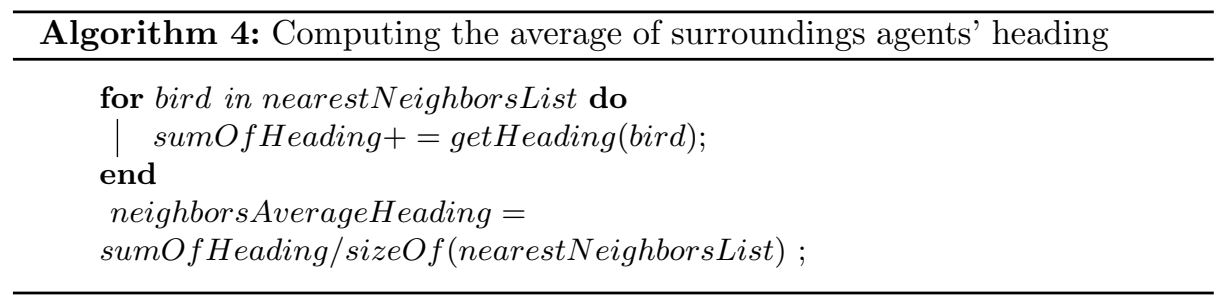

This loop is very costly when there is a large number of agents because they all perform this computation for each step of the simulation.

\subsection{Designing the Average GPU Module}

To achieve GPU translation for the previous computation, we extract information from agents' attributes (heading) and then delegate the associated computation (the loop) to the environment dynamics. To this end, for each simulation step, each agent put its heading value in a $2 \mathrm{D}$ array (headingArray (a grid matching the size of the environment) according to its position. This array is sent to a GPU module (average module) that simultaneously, for each cell within the FieldOfView, performs the average of the headings of the surrounding agents. More precisely, each thread computes the average for a cell depending on its location in the global GPU grid (its identifiers: $i$ and $j$ in Algorithm 5). The GPU translation thus consists in transforming the sequential computation previously done in the cohesion behavior of the agents into a parallel computation made on the GPU and managed by the environment. Once done, the average headings are available in every cells of the environment. So, the agents can access this data instantaneously with respect to their position (from the 2D array, flockCentering, returned by the GPU module) and then adapt their movement accordingly.

Algorithm 5 presents an implementation of the GPU average module: Once the coordinates $i$ and $j$ of the thread are initialized, the algorithm tests if the current thread's coordinates do not exceed the size of the environment (represented here by the 2D array headingArray). Next, the sum of all the headings are assigned to sumOfheading, which is then divided by the number of agents taken into account. The module then returns the array flockCentering containing all the averages.

${ }^{10}$ In the context of TurtleKit, FieldOfView is the range which is used to select the cells around the agent. 


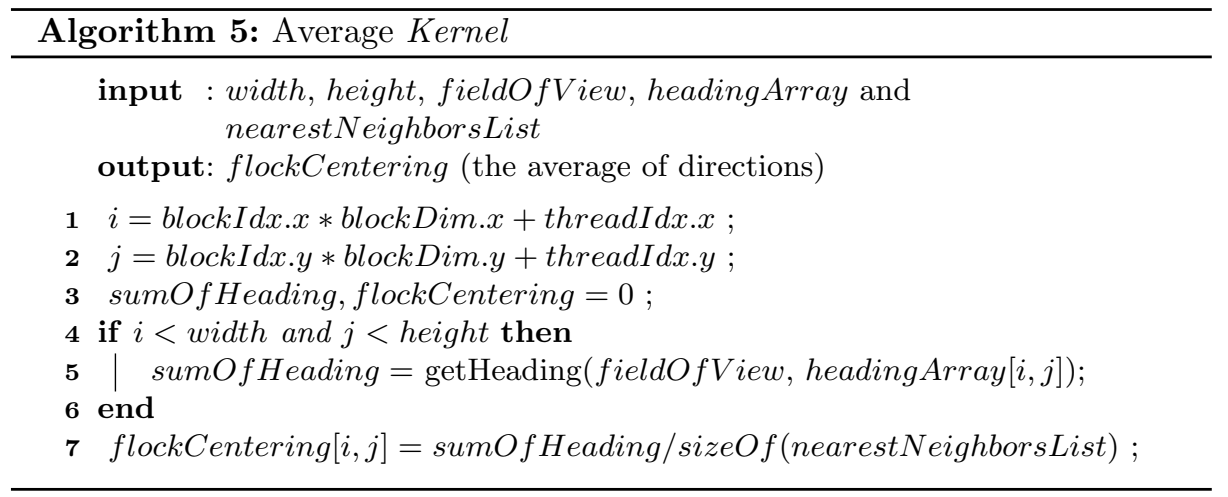

Compared with the sequential version (Algorithm 4, the loop has disappeared. So, one of the main interest of the GPU version lies in the fact that the parallelization of the loop is realized thanks to the hardware architecture, not through code parallelization: Programming with GPU, parts of the code are in the structure.

\subsection{Implementation and Integration of the GPU Average Module}

The implementation of the GPU average module has been done with CUDA and JCuda ${ }^{11}$. Figure 4 illustrates the integration of the GPU average module in TurtleKit. The implementation was easy thanks to the independence between this module and the agent model.

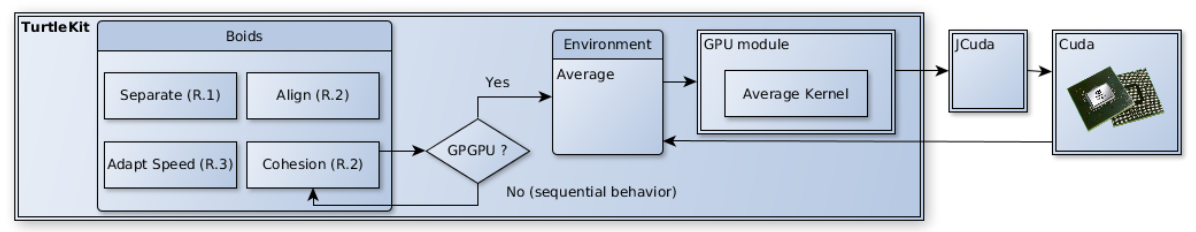

Fig. 4. Integrating the GPU Average module in TurtleKit

\section{Experimentation}

\subsection{Experimental Protocol}

To trial our implementation of Reynolds's boids and the application of GPU delegation, we simulated several environment sizes while varying the number

$\overline{11}$ The JCuda library allows to call GPU kernels, written in C, directly from Java 
of agents. We execute successively the sequential version of the model (i.e. the average is computed in the agents' behavior) then the GPGPU version (using the GPU average module). To estimate the performances according to the criteria used in section 2, we compare the average computation time in milliseconds for an iteration.

\subsection{Performance Tests}

For those tests, the configuration is identical as the one used in Sect. 2 and is composed of an Intel i7-4770 processor (Haswell generation, $3.40 \mathrm{GHz}$ ) and an Nvidia K4000 graphics card (768 CUDA cores). Figure 5 presents the results obtained for various population sizes in an $256 \times 256$ environment (top) and then in an $512 \times 512$ environment (bottom).
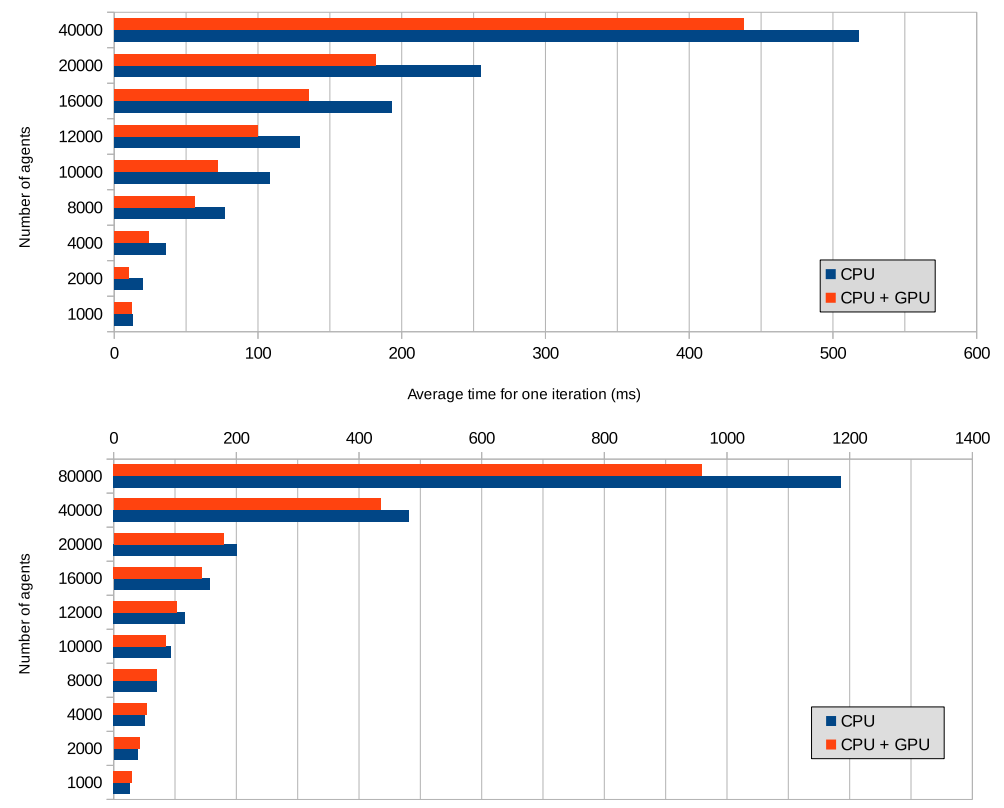

Fig. 5. Comparison of flocking simulations done with and without the GPU. Environment size: 256 (top) and 512 (bottom)

The use of the GPU module increases the performances by $25 \%$. However, we notice that the performances is linked to the density of population. Indeed, when the density of the agents in the environment is lower, agents spend fewer time in cohesion and more to align itself and to separate. The density of the agents affects performance of the model when using the GPU module. The tipping point is clearly visible in the results, when the density of present agents exceeds $5 \%$ (respectively 1500 and 8000 entities), the joint use of the CPU and the GPU 
becomes more effective. So, the more the density of agents in the environment increases, more the observed gains of performances are important.

The performance gains are interesting considering the used hardware: Our Nvidia K4000 embeds 768 CUDA cores while the last Nvidia Tesla K40 card embeds 2880 CUDA cores and the Nvidia Tesla K10 card embeds 3072 cores (two GPU with 1536 cores on the same card). The fast evolution of GPGPU and graphics cards promise very significant gains of performances in the future.

\subsection{Discussion}

In addition to the observed performance gains, we noticed other benefits related to GPU Delegation principle: Translating perception computations done in the agent behavior into environmental dynamics allows to remove a part of the source code and thus simplify the understanding of the behavior. It is more readable because the agent does not have to deal with raw data. Indeed, the agent makes a direct perception in the environment instead of a sequential computation which can be rather heavy.

Another interesting aspect is that the created modules are independent from the considered ABM thanks to this approach. They are thus not limited to the context in which they were defined. That is why we will continue to apply GPU Delegation to create new GPU modules in order to incrementally build a generic GPU modules library. This GPU library will improve the accessibility of the approach and the use of the GPGPU in the MABS context with TurtleKit. This improvement in terms of genericness and accessibility is important because working with GPGPU often leads to implementation difficulties due to the specificity of this technology.

Moreover, GPU delegation is based on a simple criterion which is independent from the implementation. So, GPU delegation allows to convert the model and create the GPU module easily in a rather fast way. TurtleKit being still in alpha release, we are going to continue to work on its architecture in order to make the conversion of a model as simple as possible.

\section{Conclusion and Perspectives}

In this paper, we described how we used the GPU Environmental Delegation of Agent Perceptions principle to implement a classic ABM, namely Reynolds's Boids, using GPGPU. Our purpose was to challenge the genericness and the ease-of-use of GPU Delegation. To this end, we needed to evolve GPU delegation so that it can be applied to the boids model. Indeed, find a computation independent from agents' attributes was impossible, so we have identified in the cohesion behavior some computations independent of agent's behaviors. We thus translated these computations into a GPU module and made some tests to see the advantages brought by the GPU Delegation.

Our experiments show that, using GPU Delegation, it is possible to increase the size of the environments and the number of agents thanks to a speed up which can reach $25 \%$ according to the chosen parameters. 
From a software engineering perspective, the use of GPU delegation allows to consider important aspects of MABS with respect to the GPGPU context. By promoting a clear separation between the agent behaviors (handled by the CPU) and environmental dynamics (managed by the GPU), GPU Delegation represents a design guideline which (1) allows to tackle the genericness issue and (2) promotes reusability of created tools. This essential criterion is often neglected in the GPGPU context [9]. GPU delegation allows the creation of generic GPU modules which are independent from the agent models.

Both implementations of the delegation principle, realized with MLE in [9] and flocking here, show that if the analysis of the model is made by keeping in mind the characteristics of the approach, the delegation of the computations and the creation of the GPU module could be very easy and fast, which is a valuable aspect of GPU Delegation, especially considering the technical difficulties related with the GPGPU context.

As GPU delegation still requires specific skills, we plan to apply to other models this principle in order to experiencing and continuing to generalize the approach. Then, as a long term perspective, our goal is to propose an explicit design methodology that would provide any MABS end user with a simple and efficient means for addressing scalability issues, without compromising reusability and accessibility which are major issues for the adoption of this technology in the MABS community.

\section{References}

1. F. Badeig, F. Balbo, and S. Pinson. A contextual environment approach for multiagent-based simulation. In ICAART'2010, 2nd International Conference on Agents and Artificial Intelligence, pages pp 212-217, Spain, June 2010. ICAART.

2. G. Beurier, O. Simonin, and J. Ferber. Model and Simulation of Multi-Level Emergence. In ISSPIT'02, Apr. 2008.

3. A. Grignard, P. Taillandier, B. Gaudou, D. Vo, N. Huynh, and A. Drogoul. GAMA 1.6: Advancing the Art of Complex Agent-Based Modeling and Simulation. In PRIMA 2013: Principles and Practice of Multi-Agent Systems, volume 8291 of Lecture Notes in Computer Science, pages 117-131. Springer Berlin, 2013.

4. Y. Kubera, P. Mathieu, and S. Picault. IODA: an interaction-oriented approach for multi-agent based simulations. Autonomous Agents and Multi-Agent Systems, 23(3):303-343, 2011.

5. G. Laville, K. Mazouzi, C. Lang, N. Marilleau, B. Herrmann, and L. Philippe. MCMAS: A Toolkit to Benefit from Many-Core Architecure in Agent-Based Simulation. In Euro-Par 2013: Parallel Processing Workshops, volume 8374 of Lecture Notes in Computer Science, pages 544-554. Springer Berlin Heidelberg, 2014.

6. G. Laville, K. Mazouzi, C. Lang, N. Marilleau, and L. Philippe. Using GPU for Multi-agent Multi-scale Simulations. In Distributed Computing and Artificial Intelligence, volume 151 of Advances in Intelligent and Soft Computing, pages 197-204. Springer Berlin Heidelberg, 2012.

7. S. Luke, C. Cioffi-Revilla, L. Panait, K. Sullivan, and G. Balan. MASON: A Multiagent Simulation Environment. Simulation, 81(7):517-527, 2005. 
8. M. Lysenko and R. M. D’Souza. A Framework for Megascale Agent Based Model Simulations on Graphics Processing Units. Journal of Artificial Societies and Social Simulation, 11(4):10, 2008.

9. F. Michel. Translating Agent Perception Computations into Environmental Processes in Multi-Agent-Based Simulations: A means for Integrating Graphics Processing Unit Programming within Usual Agent-Based Simulation Platforms. Systems Research and Behavioral Science, 30(6):703-715, 2013.

10. F. Michel, G. Beurier, and J. Ferber. The TurtleKit Simulation Platform: Application to Complex Systems. In Workshops Sessions of the Proceedings of the 1st International Conference on Signal-Image Technology and Internet-Based Systems, pages 122-128. IEEE, november 2005.

11. J. D. Owens, D. Luebke, N. Govindaraju, M. Harris, J. Kruger, A. E. Lefohn, and T. J. Purcell. A Survey of General-Purpose Computation on Graphics Hardware. Computer Graphics Forum, 26(1):80-113, 2007.

12. D. Payet, R. Courdier, N. Sébastien, and T. Ralambondrainy. Environment as support for simplification, reuse and integration of processes in spatial MAS. In Proc. of the 2006 IEEE International Conference on Information Reuse and Integration, USA, pages 127-131. IEEE Systems, Man, and Cybernetics Society, 2006.

13. M. Resnick. StarLogo: An Environment for Decentralized Modeling and Decentralized Thinking. In Conference Companion on Human Factors in Computing Systems, CHI '96, pages 11-12, New York, NY, USA, 1996. ACM.

14. C. W. Reynolds. Flocks, Herds and Schools: A Distributed Behavioral Model. In Proceedings of the 14th Annual Conference on Computer Graphics and Interactive Techniques, volume 21 of SIGGRAPH Computer Graphics '87, pages 25-34, New York, NY, USA, 1987. ACM.

15. P. Richmond, D. Walker, S. Coakley, and D. M. Romano. High performance cellular level agent-based simulation with FLAME for the GPU. Briefings in bioinformatics, 11(3):334-47, 2010.

16. Y. Sano, Y. Kadon, and N. Fukuta. A Performance Optimization Support Framework for GPU-based Traffic Simulations with Negotiating Agents. In Proceedings of the 2014 Seventh International Workshop on Agent-based Complex Automated Negotiations, 2014.

17. E. Sklar. NetLogo, a Multi-agent Simulation Environment. Artificial Life, 13(3):303-311, 2007.

18. G. Vigueras, J. Orduña, and M. Lozano. A GPU-Based Multi-agent System for Real-Time Simulations. In Advances in Practical Applications of Agents and Multiagent Systems, volume 70 of Advances in Intelligent and Soft Computing, pages 15-24. Springer Berlin Heidelberg, 2010. 